OPEN ACCESS

Edited by:

Joao P. B. Viola,

Instituto Nacional de

Câncer (INCA), Brazil

Reviewed by:

Juan J. Garcia-Vallejo,

VU University Medical

Center, Netherlands

Peter Monk,

University of Sheffield,

United Kingdom

${ }^{*}$ Correspondence:

Björn Spittau

bjoern.spittau@med.uni-rostock.de

Specialty section: This article was submitted to Molecular Innate Immunity, a section of the journal

Frontiers in Immunology

Received: 22 March 2018 Accepted: 12 July 2018

Published: 26 July 2018

Citation:

Neidert N, von Ehr A, Zöller T and Spittau B (2018) Microglia-Specific

Expression of Olfm/3 is Directly Regulated by Transforming Growth Factor $\beta 1$-Induced Smad2 Signaling.

Front. Immunol. 9:1728

doi: 10.3389/fimmu.2018.01728

\section{Microglia-Specific Expression of Olfm/3 Is Directly Regulated by Transforming Growth Factor ק1-Induced Smad2 Signaling}

\author{
Nicolas Neidert ${ }^{1}$, Alexander von Ehr ${ }^{1}$, Tanja Zöller ${ }^{1}$ and Björn Spittau ${ }^{1,2 *}$ \\ ${ }^{1}$ Department of Molecular Embryology, Faculty of Medicine, Institute for Anatomy and Cell Biology, University of Freiburg, \\ Freiburg, Germany, ${ }^{2}$ Institute of Anatomy, University of Rostock, Rostock, Germany
}

Microglia maturation takes place during the postnatal weeks and is characterized by the establishment of a unique microglia-specific gene expression pattern. Tmem119, Fcrls, Hexb, and Olfm/3 have been identified among these microglia-specific genes. Transforming growth factor $\beta 1$ (TGF $\beta 1$ ) has been reported as a critical factor for microglia maturation and maintenance and active TGF $\beta$ signaling precedes the inductions of microglial gene expression. In this study, we demonstrate Olfm/3 expression in adult microglia and further provide evidence that TGF $\beta 1$ induces upregulation of $O / f m / 3$ expression in postnatal microglia. Using chromatin immunoprecipitation and microglia-specific silencing of TGF $\beta$ signaling in vitro and in vivo, we in clearly show that Olfml3 is a direct TGF $\beta 1 / \mathrm{Smad} 2$ target gene. Together, our data underline the importance of TGF $\beta 1$ as a critical regulator of microglia functions and microglia maturation and further broaden our understanding of TGF $\beta 1$-mediated effects on the resident immune cells of the central nervous system.

Keywords: Olfml3, microglia, transforming growth factor $\beta 1$, Smad2, Smad4

\section{INTRODUCTION}

Nearly hundred years ago, Pío del Río-Hortega described microglia as the resident immune cells of the central nervous system (CNS) and already proposed a mesodermal origin of these cells in a collection of original papers, which have been made available in translated English versions (1). Novel transgenic approaches have offered a deeper insight into microglia biology and, thus, the microglia origin has just recently been elucidated in detail. In contrast to peripheral macrophages, microglia arise from hematopoietic precursors in the embryonic yolk sac dependent on PU.1 as well as Irf8 signaling pathways (2). During embryonic development, neuron-derived interleukin-34 (IL34) serves as the most potent factor to guide migrating microglia toward the CNS parenchyma (3). Ginhoux et al. (4) have identified the colony-stimulating factor-1 receptor as being essential to sense neuronal IL-34 and to mediate appropriate microglial colonization of the CNS. Next, to the unique and distinct origin, microglia further adopt a cell type-specific gene expression pattern including Olfactomedin-like 3 (Olfml3), which discriminates these CNS immune cells from other macrophage populations (5-8). The development of this microglia-specific molecular signature occurs during the first postnatal weeks in mice and includes the induction of genes such as Tmem119, Hexb, Fcrls, and $\operatorname{Tgfbr} 1(9,10)$. It is of utmost interest to understand which endogenous factors are involved in the 
induction of the microglia phenotype and the microglia maturation. However, the detailed molecular mechanisms are not well understood and based on the current knowledge transforming growth factor $\beta 1$ (TGF $\beta 1$ ) seems to be one of the most important factors. Butovsky et al. (11) have developed TGF $\beta 1$-deficient mutant mice, which presented a decrease in postnatal microglia numbers and associated with severe impairment of the establishment of the unique microglia gene expression pattern in vivo. Moreover, we have recently described that activated TGF $\beta$ signaling precedes the induction of microglia-specific gene expression and further identified the recently introduced microglia marker Tmem 119 as a direct TGF $\beta 1 / \mathrm{Smad} 2$ target gene (10). Next, to the regulation of microglia maturation, TGF $\beta 1$ has been described as a potent regulator of microglia functions by promoting microglia quiescence (12) and regulating microglia-mediated phagocytosis (13). The fact that TGF $\beta 1$ orchestrates postnatal microglia development and further regulates maintenance of adult microglia defines this versatile cytokine as an essential factor for microglia biology. To increase the understanding of TGF $\beta 1$-driven microglia maturation, we analyzed the impact of TGF $\beta$ signaling on microglial Olfml3 expression. Olfactomedinlike 3 (Olfml3) also referred to as OLF 44 has been introduced as a secreted glycoprotein (14). It is suggested that members of the Olfactomedin-like protein subfamily are involved in the development and functional organization of the CNS and the hematopoietic system (15). In this study, we provide evidence that $\mathrm{Olfm} 3 \mathrm{3}$ expression is restricted to microglia and that TGF $\beta 1$ upregulates Olfml3 expression. Using microglia-specific Tgfbr2mutant mice and chromatin immunoprecipitation (ChIP), we further demonstrate that TGF $\beta$ signaling is essential for induction of microglial Olfml3 expression and introduce $\mathrm{Olfml3}$ as direct TGF $\beta 1 /$ Smad 2 target gene. Our data increase the understanding of the molecular mechanisms that regulate microglia maturation and further strengthen the functional importance of TGF $\beta 1$ for microglia biology.

\section{MATERIALS AND METHODS}

\section{Animals}

C57BL/6JRj mice were obtained from Janvier (Le Genest Saint Isle, France) and housed at $22 \pm 2{ }^{\circ} \mathrm{C}$ under a $12 \mathrm{~h}$ light/dark cycle with ad libitum access to food and water. All animal procedures were conducted in accordance with the German federal animal welfare law, local ethical guidelines of the University Freiburg and have been approved by the animal experimentation committee of the University of Freiburg as well as the Regierungspräsidium Freiburg (G-13/57 [Tgfbr2-MG-KO], X-15/01A [primary microglia]).

\section{Microglia-Specific Tgfbr2-Knockout Mice}

The generation of $\mathrm{Cx} 3 \mathrm{cr} 1^{\mathrm{CreERT}}: \mathrm{R} 26-\mathrm{YFP}: T g f b r 2^{\mathrm{Al} / \mathrm{fl}}$ mice has been described recently (10). Briefly, mice carrying loxP-siteflanked alleles of $\mathrm{Tg} f b r 2$ were crossed to the $\mathrm{Cx} 3 \mathrm{cr} 1^{\text {CreERT2 }}$ mouse line (16). Furthermore, the reporter mouse line B6.129 × 1-Gt (ROSA)26Sortm1(EYFP)Cos/J (17) was introduced to obtain $\mathrm{Cx} 3 \mathrm{Cr} 1^{\mathrm{CreERT}}: \mathrm{R} 26-Y F P: T g f b r 2^{\mathrm{A} / \mathrm{ll}}$ mice. Cre recombinase activity was induced in vivo by treatment of 6- to 8-week-old mice with $8 \mathrm{mg}$ tamoxifen (TAM, T5648, Sigma-Aldrich, Germany) solved in $200 \mu \mathrm{l}$ corn oil (C8267, Sigma) injected intraperitoneally (two time points $48 \mathrm{~h}$ apart). Littermates carrying the respective loxP-flanked alleles but lacking expression of Cre recombinase $(+/+$ TAM) or not receiving tamoxifen (cre/+OIL) were used as controls. In vitro recombination was achieved using OH-TAM (H7904, Sigma-Aldrich, Germany) at a final concentration of $1 \mu \mathrm{M}$ to $25 \mathrm{~cm}^{2}$ glia culture flasks (single brain cultures) at least 3 days before harvesting microglia. Ethanol (EtOH) was used as a solvent control for in vitro experiments.

\section{Primary Microglia Cultures}

Primary microglia cultures were generated as described by Spittau et al. (12). Vessels and meninges were removed from brains of P0/P1 C57BL/6JRj mice (Janvier) and brains were washed and collected in ice-cold Hank's Buffered Salt Solution (Gibco, Germany). After enzymatic dissociation with Trypsin-EDTA (Gibco, Germany) for $15 \mathrm{~min}$ at $37^{\circ} \mathrm{C}$, an equal volume of fetal calf serum (FCS, Gibco, Germany) and DNase (Roche, Mannheim, Germany) at a final concentration of $0.05 \mathrm{mg} / \mathrm{ml}$ was added. Cells were dissociated using wide- and narrow-bored polished Pasteur pipettes and further centrifuged and resuspended in DMEM/F12 medium (Gibco, Germany) containing 10\% FCS and 1\% penicillin/streptomycin (Invitrogen). Dissociated cells from two to three brains were plated on poly-D-lysine-coated (Sigma-Aldrich, Schnelldorf, Germany) $75 \mathrm{~cm}^{2}$ culture flasks. Cells were kept in a $5 \% \mathrm{CO}_{2} / 95 \%$ humidified atmosphere at $37^{\circ} \mathrm{C}$. After $10-14$ days in culture, microglia were shaken off (250-300 rpm for $1 \mathrm{~h}$ ) from adherent astrocytes and plated according to the experimental designs. Treatment with recombinant human TGF $\beta 1$ (Peprotech, Hamburg, Germany) was performed at a concentration of $5 \mathrm{ng} /$ $\mathrm{ml}$. For inhibition of microglial TGF $\beta$ signaling, a TGF $\beta$ receptor type I inhibitor (\#616454, Calbiochem, Merck, Darmstadt, Germany) at a final concentration of $500 \mathrm{mM}$ was used. For inhibition of protein biosynthesis, cycloheximide (CHX) (C7698, Sigma-Aldrich, MO, USA) was used at a concentration of $50 \mu \mathrm{g} /$ $\mathrm{ml}$.

\section{BV2 Cell Culture}

BV2 cells were cultured as recently described by Zhou et al. (18). Briefly, cells were maintained in DMEM/F12 culture medium (Thermo Fisher Scientific) supplemented with 10\% FCS and $1 \%$ penicillin/streptomycin (Invitrogen) and kept in at $37^{\circ} \mathrm{C}$ in $5 \% \mathrm{CO}_{2} / 95 \%$ humidified atmosphere. Treatments with $5 \mathrm{ng} / \mathrm{ml}$ TGF $\beta 1$ (Peprotech, Hamburg, Germany) for ChIP experiments were performed under serum-free conditions.

\section{RNA Isolation and Reverse Transcription}

RNA was isolated from primary microglia using TRIzol reagent (Invitrogen, Karlsruhe, Germany) according to the manufacturer's instructions. RNA concentration and quality were determined using the NanoDrop 2000 (Thermo Scientific, Germany). $1 \mu \mathrm{g}$ total RNA from each sample was reverse transcribed to cDNA using Protoscript ${ }^{\circledR}$ II First Strand cDNA Synthesis Kit (\#E6560S, New England Biolabs, Frankfurt, Germany) according to the manufacturer's instructions. 


\section{Quantitative RT-PCR}

Quantitative RT-PCR was performed using the CFX Connect ${ }^{\mathrm{TM}}$ System (Bio-Rad, München, Germany) in combination with the SYBR Green GoTaq ${ }^{\circledR}$ qPCR Kit (A6002, Promega, Madison, WI, USA). $5 \mu \mathrm{l}$ of cDNA template was used in $20 \mu \mathrm{l}$ reaction mixture. Results were analyzed using the CFX Connect $^{\mathrm{TM}}$ System (Bio-Rad, München, Germany) Software and the comparative CT method. All data are expressed as $2^{-\triangle \Delta C T}$ for the gene of interest normalized to the housekeeping gene Gapdh and presented as fold change relative to controls. The following primers have been used throughout this study: Olfml3 for 5'-CACCTTGTGGAGTACATGGAAC-3', Olfml3 rev 5'-CTACCTCCCTTTCAAGACGGT-3' [NM_133859.2], Gapdh for 5'-GGCATTGCTCTCAATGACAA-3', Gapdh rev 5'-ATGTAGGCCATGAGGTCCAC-3' [NM_001289726], Olfml3SBE1 for 5' -TGACAGCTCTAACAGGGCCTA-3', Olfml3-SBE1 rev 5'-ACTCTGACCCCTTGAAAAGGC-3' [Chr. 3; 103739520103739508], Olfml3-SBE2 for 5' -CCCATCTCTGGTGTCTCT CAC-3', Olfml3-SBE2 rev 5'-TAGTTAAGGCTTCTGGCGACT-3' [Chr. 3; 103738043-103738055].

\section{Chromatin Immunoprecipitation}

Chromatin immunoprecipitation was performed as recently described (10). The enzymatic ChIP Kit (\#9003, Cell Signaling Technology, Inc., Danvers, MA, USA) was used according to the manufacturer's instructions. After plating of $9 \times 10^{6} \mathrm{BV} 2$ cells in $6 \times 75 \mathrm{~cm}^{2}$ cell culture flasks in DMEM/F12 medium containing 10\% FCS and 1\% penicillin/streptomycin, BV2 cells were kept $2 \mathrm{~h}$ in serum-free medium. Cells were incubated in serum-free medium containing $5 \mathrm{ng} / \mathrm{ml}$ TGF $\beta 1$. Proteins were cross-linked to the DNA by adding $1 \%$ formaldehyde to the cells for $10 \mathrm{~min}$ at room temperature (RT). The cells were harvested by scraping them into PBS + Protease inhibitor cocktail. After nuclei preparation with Buffer A and B from the kit, the nuclei from each treatment were separated into four IP samples per treatment. Digestion of chromatin was initiated by adding $0.25 \mu \mathrm{l}$ Micrococcal Nuclease (\#10011, Cell Signaling) for $20 \mathrm{~min}$ at $37^{\circ} \mathrm{C}$ and the nuclei were lysed with three sets of $20 \mathrm{~s}$ pulses using a Bioruptor $^{\mathrm{TM}}$ (Diagenode, Liège, Belgium) sonicator. Chromatin concentration of every sample was measured using NanoDrop 2000/2000c (Thermo Fisher Scientific, Waltham, MA, USA) and $10 \mu \mathrm{g}$ of digested, cross-linked chromatin of every sample was used for further steps. A $2 \%$ input control was taken aside before starting the IP. A Histone H3 antibody (Cell Signaling, \#4620, $10 \mu \mathrm{l}$ ) as a positive control, normal rabbit IgG (Cell Signaling, $\# 2729,2 \mu \mathrm{l}$ ) as a negative control and Smad2 (Cell Signaling, \#5339, 1:100) and Smad4 (Cell Signaling, \#38454, 1:100) antibodies were used for IP. The Chromatin was incubated overnight at $4^{\circ} \mathrm{C}$. After incubating the chromatin with protein $\mathrm{G}$ magnetic beads for $2 \mathrm{~h}$ and washing the chromatin in magnetic separation racks (Cell Signaling, \#7017), elution of the chromatin was performed. The protein cross-link was reversed by using $2 \mu$ Proteinase $\mathrm{K}$ (Cell Signaling, \#10012) for $2 \mathrm{~h}$ at $65^{\circ} \mathrm{C}$. Finally, DNA purification in spin columns was performed and the amount of DNA was quantified by using qPCR. Data are expressed as $2^{-\Delta C T}$ for the Smad-binding element (SBE) of interest normalized to the rabbit
IgG control. PCR products were visualized using agarose gel electrophoresis and staining with GelRed (Genaxxon Bioscience). Images were captured using a Biometra (Göttingen, Germany) gel documentation station.

\section{Immunohistochemistry and Immunocytochemistry}

Anesthetized 6-month-old C57BL/6 mice were transcardially perfused using PBS followed by $4 \%$ paraformaldehyde (PFA). Afterward, brains were extracted and postfixed in 4\% PFA overnight. Free-floating $50 \mu \mathrm{m}$ thick vibratome (Leica, Wetzlar, Germany) sections were stained overnight with anti-Ibal (1:500, Wako Chemicals, Japan) and anti-Olfml3 (1:100, sc-243668, Santa Cruz Biotechnology Inc.). Alexa Fluor-488, as well as Alexa Fluor-568-conjugated secondary antibodies (1:200, Cell Signaling Technology) was used for $2 \mathrm{~h}$ at RT. Finally, nuclei were stained using 4'6-diamidino-2-phenylindole (Dapi, Roche) for $5 \mathrm{~min}$ and sections were mounted on objective slides and covered with Fluoromount $G$ mounting medium. Immunocytochemistry was performed using PFA-fixed primary microglia on glass coverslips as mentioned above. FITC-coupled tomatolectin (Sigma-Aldrich) was used to label microglia and anti-Olfml3 (Santa Cruz Biotechnology Inc.) to detect Olfml3 expression. Localization of $\mathrm{Olfml} 3$ in the endoplasmic reticulum (ER) and the Golgi apparatus was confirmed using the Organelle Localization IF Antibody Sampler Kit (Cell Signaling technologies, \#8653). Fluorescence-coupled secondary antibodies (1:200, Cell Signaling Technologies) were used for $1 \mathrm{~h}$ at RT. Coverslips were washed three times with PBS for 3 min each and nuclei were counterstained using DAPI (Roche). After a final washing $(3 \times)$, coverslips were mounted on objective slides using Fluoromount G mounting medium (SouthernBiotech). Fluorescence images were captured using the Leica TCS SP8 confocal laser scanning microscope (Leica, Wetzlar, Germany) and the LAS AF image analysis software.

\section{Statistics}

Data are given as mean \pm SEM. Statistical differences between two groups were determined using Student's $t$-test. Multiplegroup analysis was performed using one-way ANOVA followed by Bonferroni's multiple comparison post-test. $P$-values $\leq 0.05$ were considered as statistically significant. All statistical analyses were performed using the GraphPad Prism6 software (GraphPad Software Inc., La Jolla, CA, USA).

\section{RESULTS}

\section{Olfm/3 Is Expressed in Primary Mouse Microglia and Displays a Cytoplasmic Localization}

The Olfml3 gene is located on chromosome 3 and consists of three exons. Figure 1A shows that a $180 \mathrm{bp}$ Exon 1 is separated from Exon 2 (286 bp) by Intron 1, which has a total length of $584 \mathrm{bp}$. A 288 bp Intron 2 links Exon2 with Exon 3, the latter of which has a length of 4,999 bp and includes a long 5'UTR. The protein structure of Olfml3 as depicted in Figure 1A, demonstrates the 

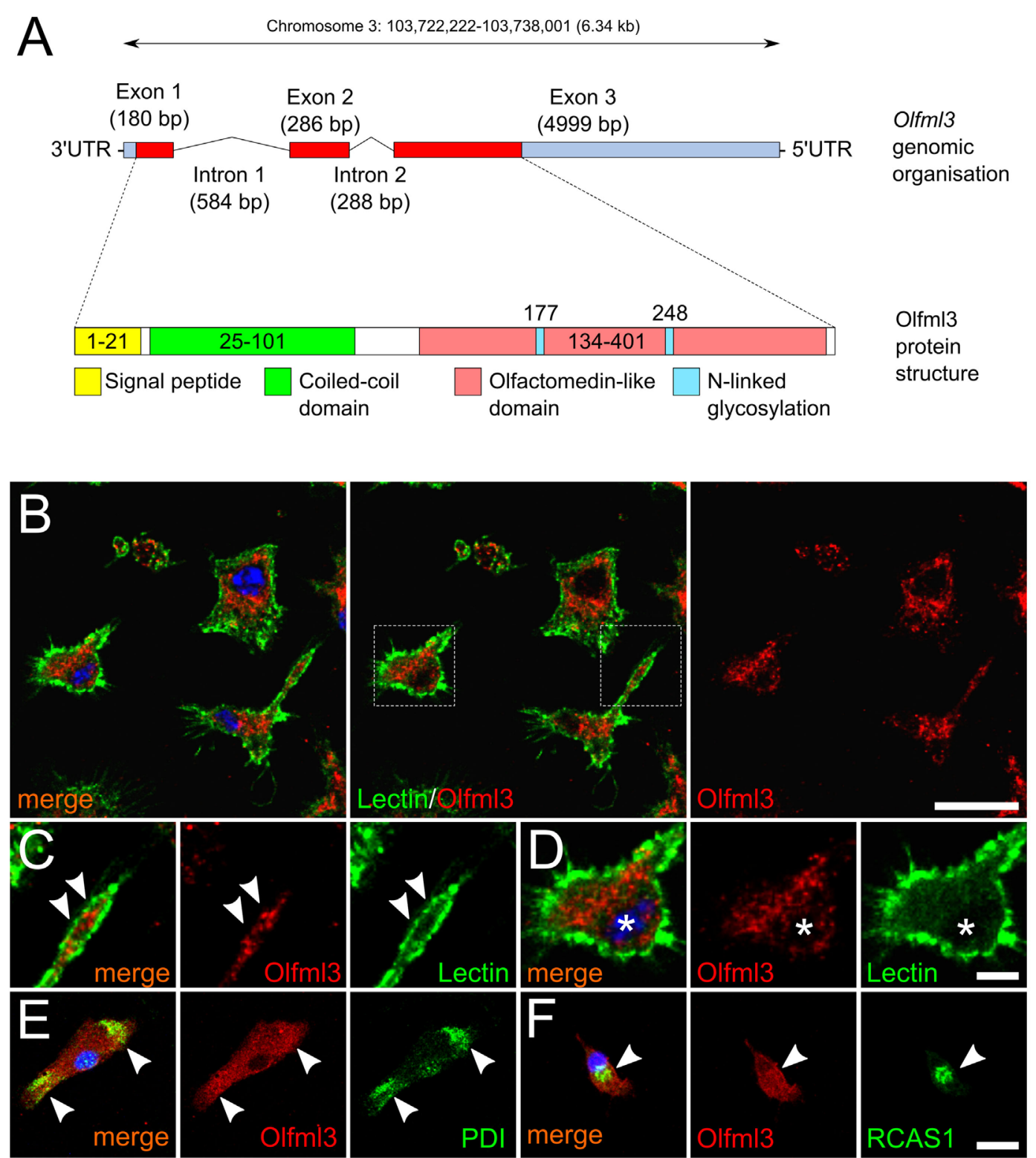

FIGURE 1 | Cytoplasmic localization of Olfml3 in primary microglia. (A) Schematic genomic organization and protein structure of Olfml3. (B) Immunofluorescence demonstrating the cellular localization of Olfml3 in primary microglia. FITC-coupled tomatolectin was used to label cell membranes. Nuclei were counterstained using DAPI. Dashed rectangles mark areas depicted in panels (C,D). Scale bar indicates $10 \mu \mathrm{m}$. (C) White arrowheads mark Olfml3 located in microglial processes.

(D) Olfml3 immunoreactivity in the perinuclear cytoplasm. Asterisks mark the nucleus. Scale bar represents $5 \mu \mathrm{m}$. (E) Colocalization of Olfml3 with the endoplasmic reticulum (ER) marker protein disulfide isomerase (PDI). White arrowheads mark Olfml3 located in the ER. (F) Colocalization of Olfml3 with the Golgi marker RCAS1.

White arrowheads mark Olfml3 located in the Golgi apparatus. Scale bar indicates $10 \mu \mathrm{m}$.

presence of 21 aa signal peptide followed by a coiled-coil domain. The central motif is the olfactomedin-like domain which harbors two N-linked glycosylation sites. We further used immunocytochemistry to detect the intracellular localization of Olfml3 in primary microglia. As shown in Figure 1B, Olfml3 immunoreactivity was found in the perinuclear cytoplasm and in microglial processes. FITC-coupled tomatolectin was used to label the cell membrane of primary microglia. Figure $\mathbf{1 C}$ displays the presence of Olfml3 speckles in microglial processes suggesting Olfml3 immunoreactivity in exocytotic vesicles. The perinuclear staining pattern of Olfml3 suggests an ER localization (Figure 1D). To confirm this subcellular localization, protein disulfide isomerase (PDI) and receptor binding cancer antigen expressed on SiSo cells (RCAS1) were used as markers for the ER and the Golgi apparatus, respectively. As shown in Figure 1E, Olfml3 colocalized with PDI indicating presence in the ER. Moreover, Olfml3 further displayed accumulation in the Golgi apparatus as evidenced by colocalization with RCAS1 (Figure 1F). Together, these data 
demonstrate that primary microglia show robust expression of the secreted glycoprotein Olfml3.

\section{Olfml3 Is Expressed in Microglia In Vivo}

It has been described that Olfml3 belongs to a set of genes, which have been demonstrated to be microglia-specific and, which are not expressed by other macrophage populations $(5,6$, $8,9)$. Since these data were obtained from RNAseq and/or gene expression studies, we used immunohistochemistry to determine Olfml3 expression in cortical microglia of adult mice. As shown in Figure 2, Olfml3 immunoreactivity could be detected in the molecular layer and the external granule cell layer of the frontal cortex (Figure 2A). Iba1 ${ }^{+}$cortical microglia (Figure 2B) were also positive for Olfml3 (Figure 2C). High magnification images reveal that strong Olfml3 immunoreactivity was detectable in the cytoplasm of $\mathrm{Iba1}^{+}$microglia (Figures 2D-F). Interestingly, microglial processes only displayed weak Olfml3 signals and Olfml3 speckles could be found in the periphery of microglia suggesting an extracellular localization and accumulation (Figure 2F). Ibal $^{+}$meningeal macrophages can be found in close proximity to the pial surface and, thus, Olfml3 immunoreactivty in this non-microglial cell was analyzed. As depicted in Figures 2G-I, Iba1 ${ }^{+}$meningeal macrophages (white arrows) showed no or very faint Olfml3 immunoreactivity whereas Iba ${ }^{+}$ cortical microglia (white asterisks) show strong Olfml3 expression. These data clearly show that Olfml3 is predominantly expressed by $\mathrm{Iba}^{+}$microglia and further suggest that $\mathrm{Olfml} 3$ is secreted and accumulated in the extracellular space.

\section{Microglial Olfml3 Expression Is Dependent on TGF $\beta$ Signaling}

It has recently been described that TGF $\beta 1$ is necessary to induce the expression of microglia-specific genes such as Olfml3 (11). Moreover, we have demonstrated that microglial TGF $\beta$ signaling at $\mathrm{P} 7$ precedes the upregulation of microglia-specific genes in vivo (10). To address whether the Olfml3 expression in microglia is dependent on TGF $\beta$ signaling, we used microglia from $C x 3 c r 1^{C r e E R T 2}: T g f b r 2^{f / f l}$ mice. As displayed in Figure 3A, 2-month-old $C x 3 \mathrm{cr} 1^{\mathrm{CreERT2}}: \mathrm{Tg} f b r 2^{f / f l}$ mice were used to generate the tamoxifen-induced microglia-specific deletion of ligandbinding receptor Tgfbr 2 in vivo. Four weeks after recombination, microglial RNA was isolated and used to detect Olfml 3 expression. Figure 3B shows that silencing of TGF $\beta$ signaling in adult microglia did not impair Olfml3 expression. Moreover, normal Olfml3
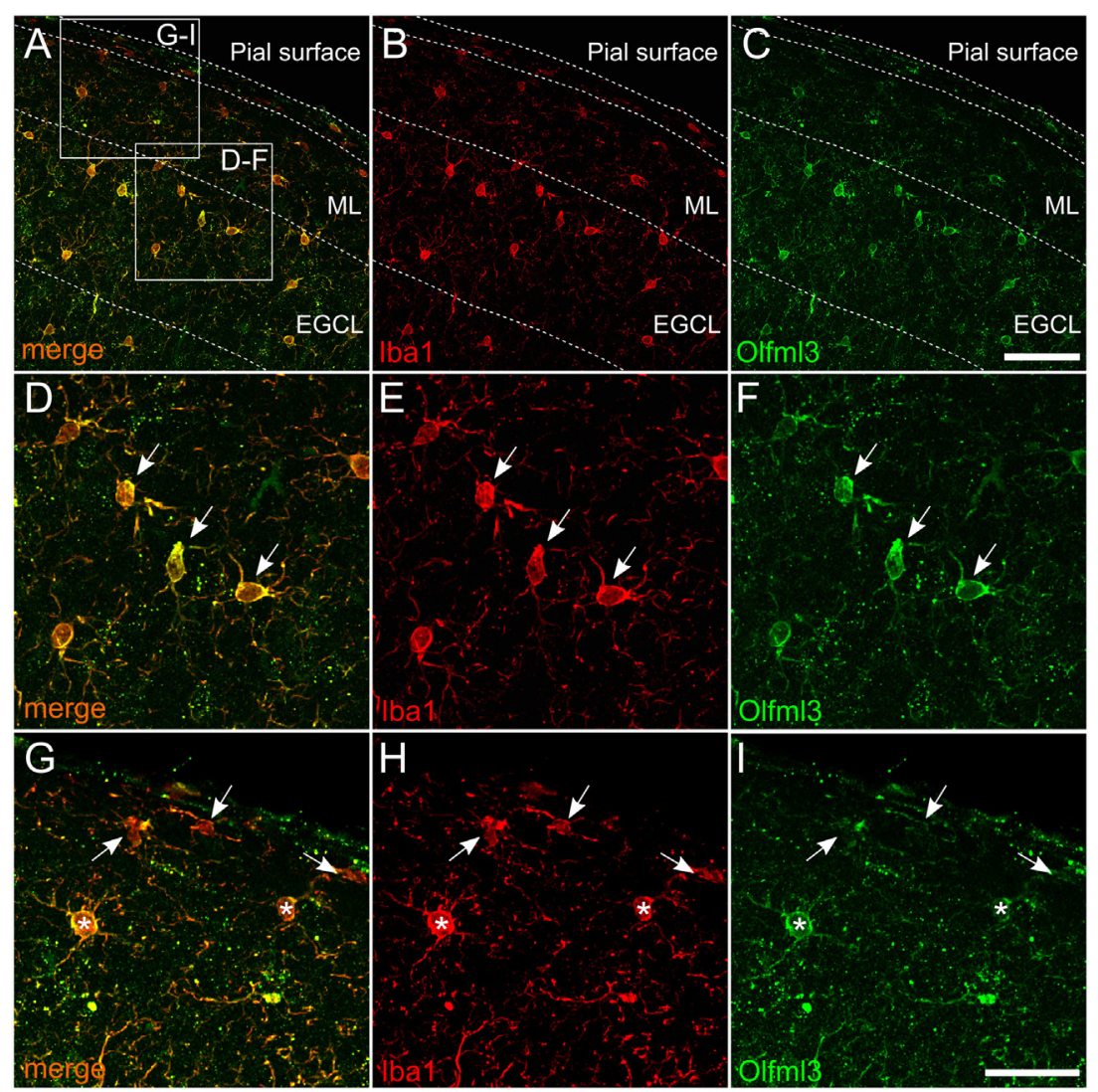

FIGURE 2 | Expression of Olfm/3 in cortical microglia. Overlay (A) of lba1+ $\mathbf{( B )}$ and Olfml3 ${ }^{+} \mathbf{~ ( C ) ~ c o r t i c a l ~ m i c r o g l i a ~ f r o m ~ 6 - m o n t h - o l d ~ C 5 7 B L / 6 ~ m i c e ~ d e m o n s t r a t e ~}$ microglia-specific expression of Olfml3. White arrows in high magnification images show strong cytoplasmic immunoreactivity for Olfml3 (F) in Iba1+ microglia (D,E). Noteworthy, Iba1+ meningeal macrophages (white arrows) located in close proximity to the pial surface show faint Olfml3 expression as compared to microglia (white asterisks) of the ML (G-I). Abbreviations: ML, molecular layer; EGCL, external granule cell layer. Scale bars represent 20 um (A-C) and $10 \mu m$ (D-I). 


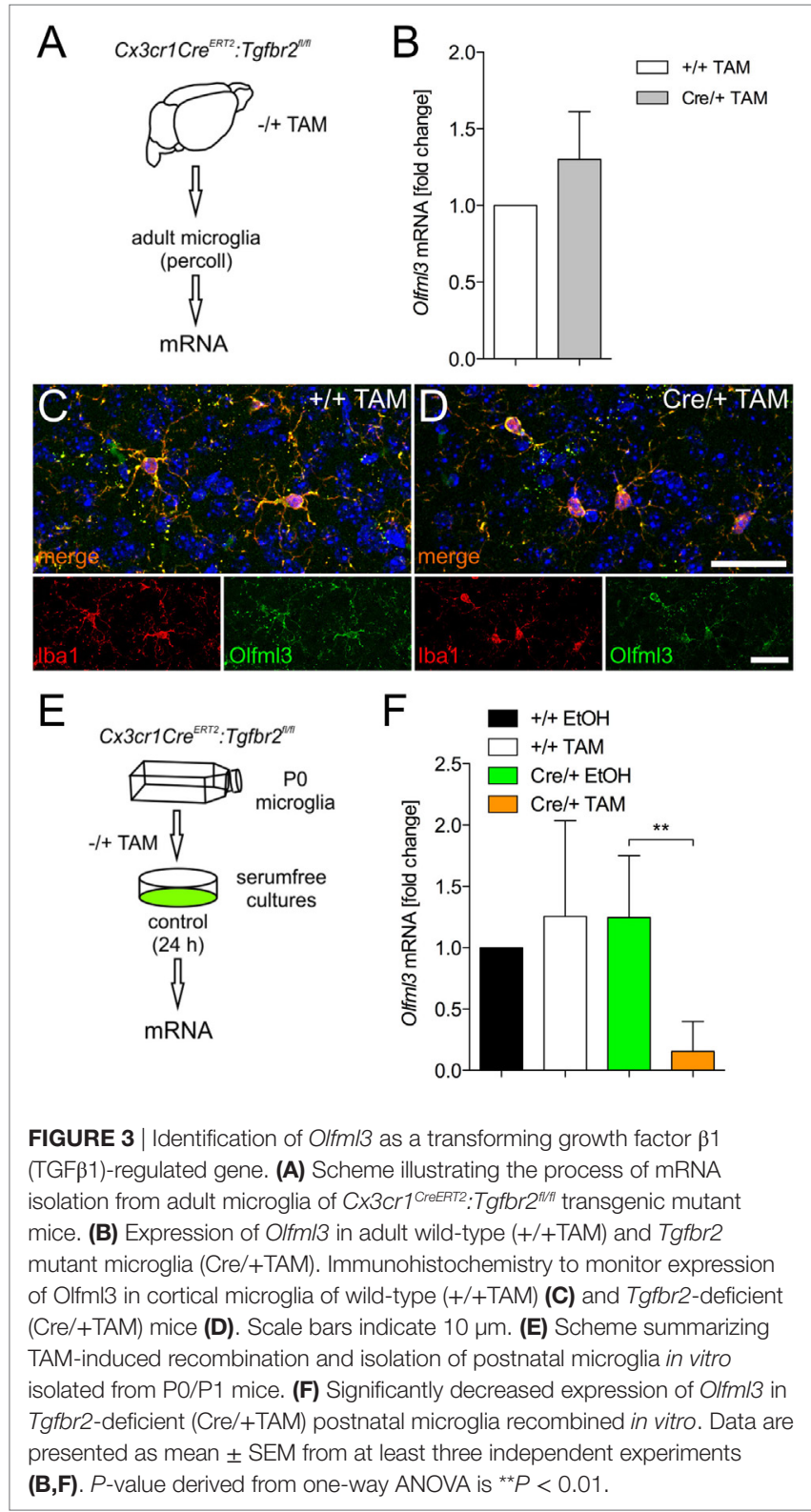

expression was observed in cortical $\mathrm{Iba}^{+}$microglia after deletion of Tgfbr2 in vivo (Figure 3D) when compared with wild-type microglia (Figure 3C). Using postnatal microglia cultures from $C \times 3 \mathrm{Cr}^{\mathrm{CrEERT2}}: \mathrm{Tg} f b r 2^{f / f l}$ mice and tamoxifen-induced recombination in vitro (Figure 3E), we observed that $\mathrm{Olfml} 3$ expression was significantly reduced in Tgfbr2-deficient microglia (Figure 3F). In summary, the presented data indicate that TGF $\beta$ signaling is essential to induce Olfml3 expression in immature postnatal microglia but is dispensable to maintain Olfml3 expression in mature adult microglia. The results further suggest that Olfml3 might be a direct TGF $\beta 1$ target gene.

\section{Identification of Olfm/3 as a Direct TGF/1/ Smad2 Target Gene}

To address whether Olfml3 is direct TGF $\beta 1$ target gene, primary microglia were treated with recombinant human TGF $\beta 1(5 \mathrm{ng} / \mathrm{ml})$ for 2, 6, and $24 \mathrm{~h}$. As depicted in Figure 4A, significant upregulation of Olfml 3 expression was detected after 2 and $6 \mathrm{~h}$. Although Olfml 3 expression was increased after 24 h of TGF $\beta 1$ treatment, no significant changes were detectable. Next, immunocytochemistry was employed to monitor increases in Olfml3 expression in primary microglia. Whereas a distinct but weak immunoreactivity for Olfml3 could be observed in untreated microglia (Figure 4B) strong increases in $\mathrm{Olfml} 3$ signals were detectable after treatment of primary microglia with TGF $\beta 1$ for $24 \mathrm{~h}$ (Figure 4C). Using CHX-mediated inhibition of protein synthesis revealed that TGF $\beta 1$-induced Olfml3 expression was independent on de novo synthesis of proteins (Figure 4D) indicating a direct upregulation of Olfml 3 transcription by activated TGF $\beta$ signaling. In silico analysis of the $\mathrm{Olfml} 3$ promoter region revealed the presence of two putative SBEs upstream of the transcriptional start site. Both SBEs contain the typical palindromic CAGAC DNA-binding sequence for Smads (Figure 4E). BV2 cells were treated with TGF $\beta 1$ for $2 \mathrm{~h}$ and ChIP was performed using Smad2- as well as Smad4-specific antibodies. Figures 4F,G show that Smad2 interacted with both putative SBEs whereas Smad4 did not. Precipitation of Histone 3 was used as the positive control for all performed ChIP experiments. These data clearly demonstrate that Olfml3 is a direct TGF $\beta 1 / \mathrm{Smad} 2$ target in microglia and further show that Smad4 is not interacting with SBEs of the Olfml3 promoter.

\section{DISCUSSION}

In this study, we have demonstrated that TGF $\beta 1$ induces the expression of the microglia-specific gene Olfml3 in primary microglia. Moreover, we provide evidence that $\mathrm{Olfml3}$ is a direct TGF $\beta 1 /$ Smad2 target gene and further revealed that postnatal microglial TGF $\beta$ signaling is essential for Olfml3 upregulation whereas lack of TGF $\beta 1$ signal transduction is dispensable for the maintenance of microglial Olfml3 expression in vivo.

We have recently demonstrated that microglial TGF $\beta$ signaling is activated at postnatal day 7 (P7) and, thus, precedes the establishment of the microglial gene expression pattern (10). Using ChIP, we identified Olfml3 as a direct Smad2 target gene. Interestingly, Smad4 could not be demonstrated to sufficiently bind the SBEs of the Olfml3 promoter. This observation is in congruence with the recently reported activation of Tmem119 expression in microglia (10) and raises the question to which extent Smad4 is necessary for TGF $\beta 1$-mediated regulation of microglial gene expression. The canonical TGF $\beta$ signaling pathway has been described to involve the TGF $\beta$-induced formation of hetero-oligomeric complexes of Tgfbr2 and Tgfbr1 (19) followed by Tgfbr1-induced phosphorylation of Smad2 at serine residues 465 and 467, which is the prerequisite for Smad2-Smad4 complex formation and subsequent nuclear translocation (20). In this study, Smad4 was not involved in Olfml3 expression and it remains to be established whether Smad4 is needed for TGF $\beta 1$-induced effects in microglia. It has been described that Smad2 is able to directly interact with nucleoporins Nup214 and Nup153 to undergo Smad4-independent nuclear translocation (21). This displays a potential mechanism to explain the observed Smad2-dependent regulation of Olfml3 expression in microglia. 

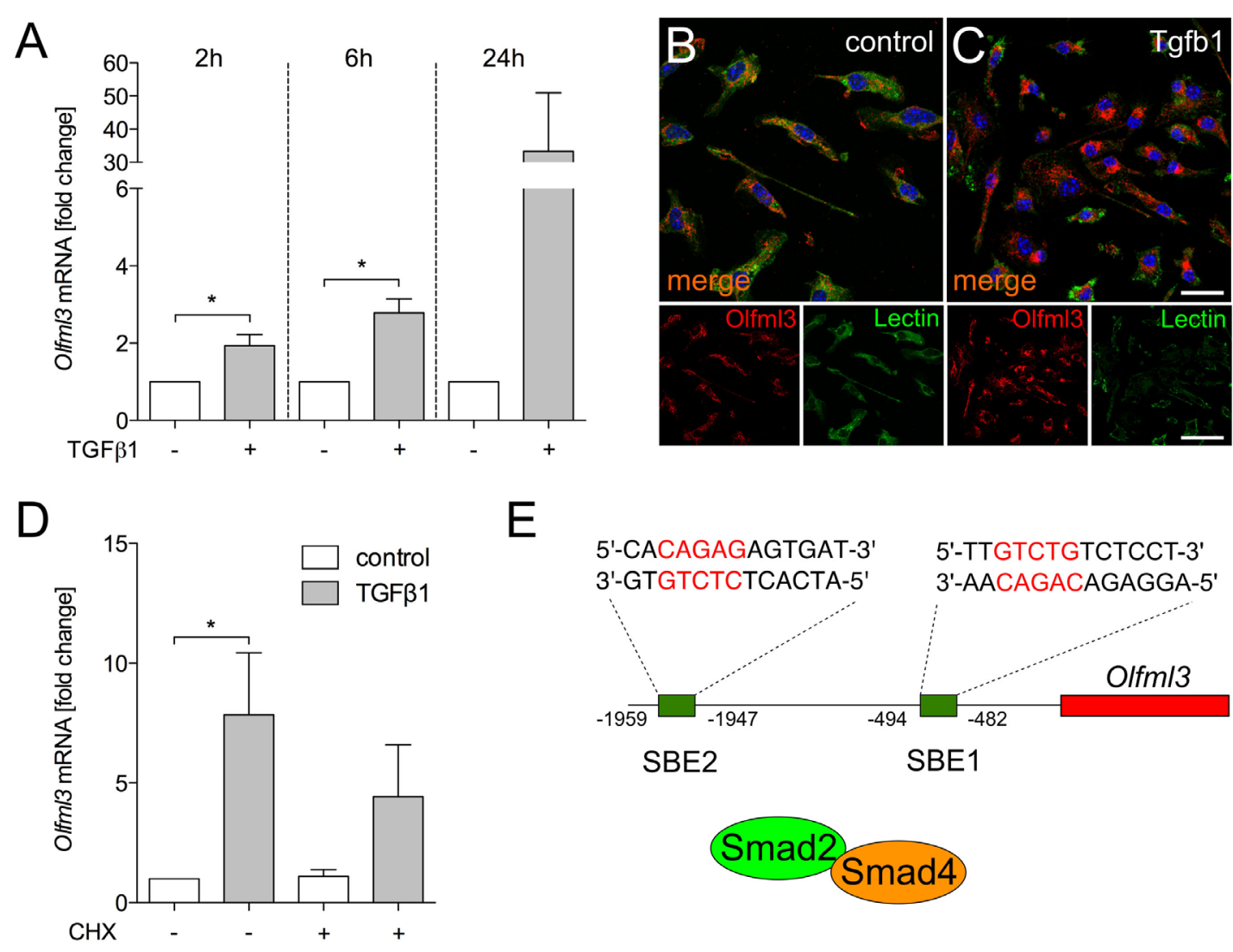

E

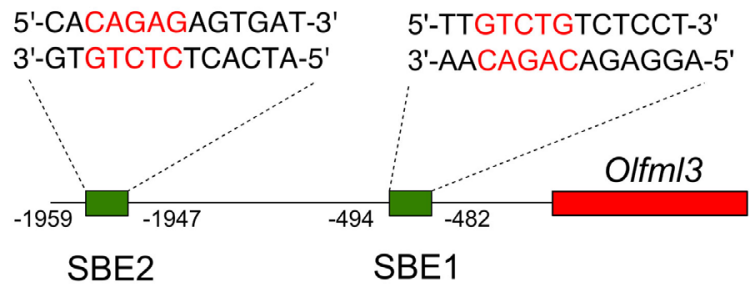

$\mathrm{F}$

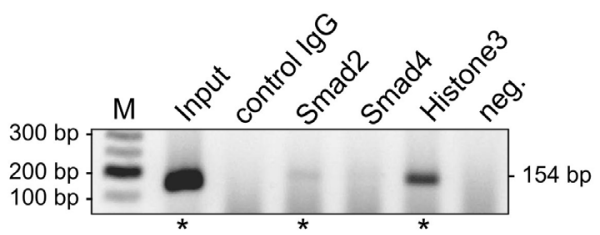

G

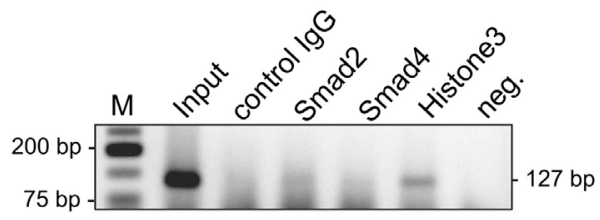

Olfm/3 Smad binding element 1

Olfm/3 Smad binding element 2
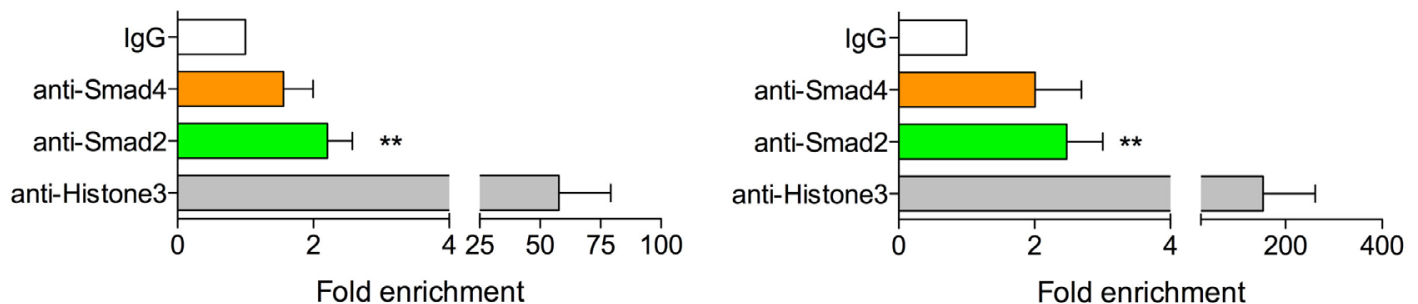

FIGURE 4 | Identification of Olfm/3 as a direct transforming growth factor $\beta 1$ (TGF $\beta 1$ )/Smad2 target gene (A) Increased expression of Olfm/3 in primary microglia treated with recombinant TGF $\beta 1(5 \mathrm{ng} / \mathrm{ml})$ for indicated time points. Immunocytochemistry demonstrating expression of Olfml3 in control (B) and TGF treated primary microglia (C) after $24 \mathrm{~h}$. FITC-coupled tomatolectin was used to label microglia. Scale bars represent $10 \mu \mathrm{m}$ for merged images and $20 \mu \mathrm{m}$ for single stain images. (D) Cycloheximide-induced inhibition of protein synthesis only marginally affected TGF $\beta 1$-induced expression of Olfm/3 in primary microglia. (E) Genomic organization of Olfm/3 displaying localization and sequence of in silico-predicted Smad-binding elements (SBEs). Results of chromatin immunoprecipitation (ChIP) PCR amplification and quantifications of qPCR results after ChIP of TGF $\beta 1$-treated BV2 microglia using Smad4- and Smad2-specific antibodies. Significant enrichment after Smad2 precipitation was detected for SBE1 (F) as well as for SBE2 (G). Asterisks mark Olfml3 promoter PCR product separated by agarose gel electrophoresis (F,G). Smad4 binding could not be observed for all SBEs. Anti-Histone3 antibodies were used as a positive control and non-specific isotype IgGs were used as a negative control for all ChIP experiments. Data are presented as mean \pm SEM from at least three independent experiments. $P$-values derived from Student's $t$-test are ${ }^{\star} P<0.05$ (A,F,G). $P$-values derived from one-way ANOVA are ${ }^{\star} P<0.05$ (D). 
However, further sophisticated studies are necessary to address the Smad2-dependent and Smad4-independent TGF $\beta 1$ effects in microglia.

Using microglia-specific deletion of microglial Tgfbr2, we demonstrated that postnatal microglia showed a significant reduction in Olfml3 expression, which further supports the notion that TGF $\beta 1$ is a critical factor to induce maturation of microglia at early postnatal stages. Noteworthy, this reduction in Olfml3 expression was not detectable when TGF $\beta 1$ signaling was abrogated in adult microglia in vivo. Here, normal microglial Olfml3 expression was observed in mutant mice indicating that intact TGF $\beta$ signaling is dispensable for maintenance of Olfml3 expression. Most studies reporting microglia-specific Olfml3 expression have used mRNA-based expression data $(8,9,11)$ and, thus, the protein distribution of Olfml3 was unknown. Here, we present data demonstrating cytoplasmic Olfml3 protein localization in vitro and in vivo. Moreover, the observed speckled in vivo staining pattern (Figure 2) suggests the extracellular accumulation of secreted Olfml3. It remains unclear what physiological functions are linked to microglia-derived Olfml3 in vivo. In this study, we have further demonstrated that Olfml3 expression in the CNS is restricted to microglia, whereas meningeal macrophages and other neural cells including neurons, astroglia, and oligodendroglia did not show Olfml3 expression. However, abundant mRNA levels of human OLFML3 (hOLF44) have been described to be detectable in the placenta, liver, and heart (14). Moreover, genetic disruption of Olfml3 in mice using a LacZknock-in strategy further revealed expression of Olfml3 during early embryogenesis in the allantois, lateral plate mesoderm as well as in the heart and the CNS. Interestingly, the homozygous mutant mice were viable and fertile and no obvious morphological phenotype has been reported (22). Nevertheless, the structural similarity to other olfactomedin proteins implies also related functions of Olfml3. The conserved olfactomedin-like domain, which is thought to mediate protein-protein interactions, has been described in organisms ranging from nematodes to humans (23). For instance, zebrafish olfactomedin 1 (Olfm1) regulates retinal axon elongation (24) by interacting with the Nogo A receptor and, thus, preventing the collapse of the growth

\section{REFERENCES}

1. Sierra A, de Castro F, Del Río-Hortega J, Rafael Iglesias-Rozas J, Garrosa M, Kettenmann H. The "Big-Bang" for modern glial biology: translation and comments on Pío del Río-Hortega 1919 series of papers on microglia. Glia (2016) 64:1801-40. doi:10.1002/glia.23046

2. Kierdorf K, Erny D, Goldmann T, Sander V, Schulz C, Perdiguero EG, et al. Microglia emerge from erythromyeloid precursors via Pu.1- and Irf8dependent pathways. Nat Neurosci (2013) 16:273-80. doi:10.1038/nn.3318

3. Greter M, Lelios I, Pelczar P, Hoeffel G, Price J, Leboeuf M, et al. Stroma-derived interleukin-34 controls the development and maintenance of Langerhans cells and the maintenance of microglia. Immunity (2012) 37:1050-60. doi:10.1016/ j.immuni.2012.11.001

4. Ginhoux F, Greter M, Leboeuf M, Nandi S, See P, Gokhan S, et al. Fate mapping analysis reveals that adult microglia derive from primitive macrophages. Science (2010) 330:841-5. doi:10.1126/science.1194637

5. Chiu IM, Morimoto ETA, Goodarzi H, Liao JT, O'Keeffe S, Phatnani HP, et al. A neurodegeneration-specific gene-expression signature of acutely isolated cone (25). Furthermore, deletion of Olfm1 in mice resulted in structural and functional impairments of white matter tracts and the olfactory system (26). In contrast to Olfm1, genetic targeting of Olfm2 did not result in gross abnormalities but mutant mice presented with abnormal locomotor coordination, reduced exploration and anxiety-related behavior (27). These studies underline the importance of olfactomedins for neurodevelopment and neuronal functions and suggest a contribution of Olfml3 in developmental and functional aspects of the postnatal CNS. Taken together, our results introduce Olfml3 as a new TGF $\beta 1 /$ Smad2 target gene in microglia and, thereby, broaden our understanding of TGF $\beta 1$-mediated regulations of microglia maturation. The role of microglia-derived Olfml3 remains elusive and further studies are necessary to elucidate the importance of microglia-derived Olfml3.

\section{ETHICS STATEMENT}

This study was carried out in accordance with the recommendations of the German federal animal welfare law and local ethical guidelines of the University Freiburg. The protocol was approved by the Regierungspräsidium Freiburg.

\section{AUTHOR CONTRIBUTIONS}

BS conceived the project and wrote the manuscript. NN, AE, TZ, and BS performed experiments and analyzed the data. All the authors have read and approved the final manuscript and further agreed to be accountable for the content of the work.

\section{ACKNOWLEDGMENTS}

The authors thank Ludmila Butenko for her excellent technical assistance.

\section{FUNDING}

This work was funded by grants from the Deutsche Forschungsgemeinschaft (DFG, SP 1555/2-1). microglia from an amyotrophic lateral sclerosis mouse model. Cell Rep (2013) 4:385-401. doi:10.1016/j.celrep.2013.06.018

6. Gautier EL, Shay T, Miller J, Greter M, Jakubzick C, Ivanov S, et al. Geneexpression profiles and transcriptional regulatory pathways that underlie the identity and diversity of mouse tissue macrophages. Nat Immunol (2012) 13:1118-28. doi:10.1038/ni.2419

7. Beutner C, Linnartz-Gerlach B, Schmidt SV, Beyer M, Mallmann MR, Staratschek-Jox A, et al. Unique transcriptome signature of mouse microglia. Glia (2013) 61:1429-42. doi:10.1002/glia.22524

8. Hickman SE, Kingery ND, Ohsumi TK, Borowsky ML, Wang L-C, Means TK, et al. The microglial sensome revealed by direct RNA sequencing. Nat Neurosci (2013) 16:1896-905. doi:10.1038/nn.3554

9. Bennett ML, Bennett FC, Liddelow SA, Ajami B, Zamanian JL, Fernhoff NB, et al. New tools for studying microglia in the mouse and human CNS. Proc Natl Acad Sci U S A (2016) 113:E1738-46. doi:10.1073/pnas.1525528113

10. Attaai A, Neidert N, von Ehr A, Potru PS, Zöller T, Spittau B. Postnatal maturation of microglia is associated with alternative activation and activated TGF $\beta$ signaling. Glia (2018) 66:1695-708. doi:10.1002/glia.23332 
11. Butovsky O, Jedrychowski MP, Moore CS, Cialic R, Lanser AJ, Gabriely G, et al. Identification of a unique TGF- $\beta$-dependent molecular and functional signature in microglia. Nat Neurosci (2014) 17:131-43. doi:10.1038/ nn0914-1286d

12. Spittau B, Wullkopf L, Zhou X, Rilka J, Pfeifer D, Krieglstein K. Endogenous transforming growth factor-beta promotes quiescence of primary microglia in vitro. Glia (2013) 61:287-300. doi:10.1002/glia.22435

13. Spittau B, Rilka J, Steinfath E, Zöller T, Krieglstein K. TGF $\beta 1$ increases microglia-mediated engulfment of apoptotic cells via upregulation of the milk fat globule-EGF factor 8. Glia (2015) 63:142-53. doi:10.1002/glia.22740

14. Zeng L-C, Liu F, Zhang X, Zhu Z-D, Wang Z-Q, Han Z-G, et al. hOLF44, a secreted glycoprotein with distinct expression pattern, belongs to an uncharacterized olfactomedin-like subfamily newly identified by phylogenetic analysis. FEBS Lett (2004) 571:74-80. doi:10.1016/j.febslet.2004.06.059

15. Anholt RRH. Olfactomedin proteins: central players in development and disease. Front Cell Dev Biol (2014) 2:6. doi:10.3389/fcell.2014.00006

16. Goldmann T, Wieghofer P, Müller PF, Wolf Y, Varol D, Yona S, et al. A new type of microglia gene targeting shows TAK1 to be pivotal in CNS autoimmune inflammation. Nat Neurosci (2013) 16:1618-26. doi:10.1038/nn.3531

17. Srinivas S, Watanabe T, Lin CS, William CM, Tanabe Y, Jessell TM, et al. Cre reporter strains produced by targeted insertion of EYFP and ECFP into the ROSA26 locus. BMC Dev Biol (2001) 1:4. doi:10.1186/1471-213X-1-4

18. Zhou X, Spittau B, Krieglstein K. TGF $\beta$ signalling plays an important role in IL4-induced alternative activation of microglia. J Neuroinflammation (2012) 9:210. doi:10.1186/1742-2094-9-210

19. Yamashita H, ten Dijke P, Franzén P, Miyazono K, Heldin CH. Formation of hetero-oligomeric complexes of type I and type II receptors for transforming growth factor-beta. J Biol Chem (1994) 269:20172-8.

20. Abdollah S, Macías-Silva M, Tsukazaki T, Hayashi H, Attisano L, Wrana JL. TbetaRI phosphorylation of Smad2 on Ser465 and Ser467 is required for Smad2-Smad4 complex formation and signaling. J Biol Chem (1997) 272: 27678-85. doi:10.1074/jbc.272.44.27678

21. Xu L, Kang Y, Cöl S, Massagué J. Smad2 nucleocytoplasmic shuttling by nucleoporins CAN/Nup214 and Nup153 feeds TGFbeta signaling complexes in the cytoplasm and nucleus. Mol Cell (2002) 10:271-82. doi:10.1016/ S1097-2765(02)00586-5
22. Ikeya M, Kawada M, Nakazawa Y, Sakuragi M, Sasai N, Ueno M, et al. Gene disruption/knock-in analysis of mONT3: vector construction by employing both in vivo and in vitro recombinations. Int J Dev Biol (2005) 49:807-23. doi:10.1387/ijdb.051975mi

23. Zeng L-C, Han Z-G, Ma W-J. Elucidation of subfamily segregation and intramolecular coevolution of the olfactomedin-like proteins by comprehensive phylogenetic analysis and gene expression pattern assessment. FEBS Lett (2005) 579:5443-53. doi:10.1016/j.febslet.2005.08.064

24. Nakaya N, Lee H-S, Takada Y, Tzchori I, Tomarev SI. Zebrafish olfactomedin 1 regulates retinal axon elongation in vivo and is a modulator of Wnt signaling pathway. JNeurosci (2008) 28:7900-10. doi:10.1523/ JNEUROSCI.0617-08.2008

25. Nakaya N, Sultana A, Lee H-S, Tomarev SI. Olfactomedin 1 interacts with the Nogo A receptor complex to regulate axon growth. J Biol Chem (2012) 287:37171-84. doi:10.1074/jbc.M112.389916

26. Nakaya N, Sultana A, Munasinghe J, Cheng A, Mattson MP, Tomarev SI. Deletion in the $\mathrm{N}$-terminal half of olfactomedin 1 modifies its interaction with synaptic proteins and causes brain dystrophy and abnormal behavior in mice. Exp Neurol (2013) 250:205-18. doi:10.1016/j.expneurol.2013.09.019

27. Sultana A, Nakaya N, Dong L, Abu-Asab M, Qian H, Tomarev SI. Deletion of olfactomedin 2 induces changes in the AMPA receptor complex and impairs visual, olfactory, and motor functions in mice. Exp Neurol (2014) 261:802-11. doi:10.1016/j.expneurol.2014.09.002

Conflict of Interest Statement: The authors declare that the research was conducted in the absence of any commercial or financial relationships that could be construed as a potential conflict of interest.

Copyright () 2018 Neidert, von Ehr, Zöller and Spittau. This is an open-access article distributed under the terms of the Creative Commons Attribution License (CC BY). The use, distribution or reproduction in other forums is permitted, provided the original author(s) and the copyright owner(s) are credited and that the original publication in this journal is cited, in accordance with accepted academic practice. No use, distribution or reproduction is permitted which does not comply with these terms. 